\title{
Haemolysis in Centrifugal Pump of Artificial Blood Circulation System
}

\author{
Bobkov A.V.* \\ Aircraft Building Faculty, Komsomolsk-on-Amur State University, Komsomolsk-on-Amur, Russia \\ *Corresponding author. Email: bobkov@knastu.ru
}

\begin{abstract}
The article deals with possible causes of increased haemolysis in the centrifugal pump of the artificial blood circulation systems. One of the hydrodynamic reasons is indicated - a strong vortex formation of the blood flow inside the pump impeller, which occurs when the impeller rotates. This factor is a natural demonstration of the mechanism of converting the mechanical energy of the impeller rotation into the pressure of the pumped blood flow.
\end{abstract}

\section{Keywords: artificial blood circulation system, centrifugal pump, haemolysis}

\section{INTRODUCTION}

Haemolysis is the destruction of the coat of red blood cells or red corpuscles which transfer oxygen to the cells of the body. The transfer is carried out by a complex red protein or haemoglobin which is contained in the cytoplasm of erythrocytes. The geometric structure of the erythrocytes that is the biconcave disk shape of to 10 microns in diameter provides free passage of these cells through the vascular system, including capillaries, to the cells of the body. This structure provides the delivery oxygen to them and the intake of carbon dioxide.

Natural physiological haemolysis occurs in every living thing, after about 120 days.

Due to the development of Artificial Blood Circulation System (ABCS), the problem of mechanical haemolysis in the ABCS appeared [1], particularly, in their rotary pumps: axial, centrifugal and disk-shaped ones, see Fig. 1. The purpose of ABCS pumps, as well as the function of the heart, is to create the pressure (to increase the static blood pressure) at the outlet that is equal to the hydraulic resistance of the human vascular system [2, 3].

Rotary pumps belong to the group of dynamic pumps. The working principle is to convert the mechanical energy of the pump rotor spinning into the hydraulic energy of the pumped liquid. This principle differs from the mechanism of the natural human heart, which can be classified as a volumetric pump. The process to convert energy in volumetric pumps is aimed at increasing the static pressure of the pumped liquid due to the mechanical energy of compression (volume reduction) of the working cavity (for the heart, this is the contraction of its right and left ventricles).

\section{Covering}
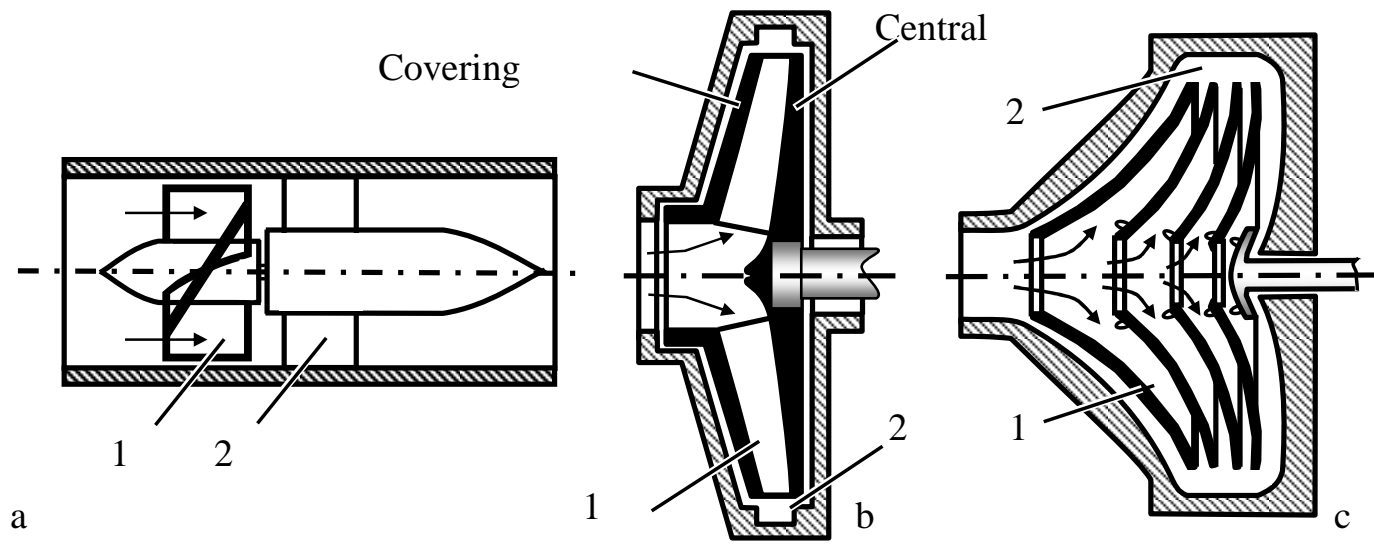

Figure 1 Rotary pumps: a - axial flow pump; b - centrifugal pump; c - pump with disc-shaped cavity. 1 - impeller; 2 discharge device

From the kinematic point of view, rotary pumps differ from volumetric ones in that the energy conversion takes place in the rotating impeller (RI) and is accompanied by a considerable increase in kinetic energy, i.e. the blood flow 
rate. At the output of the RI, in the discharge device, the kinetic energy is converted into potential energy (static pressure).

In volumetric pumps there is no impeller and the energy conversion takes place without increasing the flow rate.

The studies of hemolysis in rotary pumps of ABCS showed that the greatest damage to the blood is observed in centrifugal pumps [1]. At the same time, the authors point out that the damage is mechanical and is caused by direct contact between the blood elements and the pump rotor due to the lack of boundary layers in the rotor. However, in the papers devoted to the studies of flows in the rotors of centrifugal pumps and compressors used, in particular, in aerospace engineering, it is indicated that boundary layers exist in the inter-vane space of the impellers $[4,5]$.

It becomes relevant to analyze the characteristics of the flow in the RI centrifugal pumps to identify the causes of increased haemolysis.

\section{THE THEORETICAL MODEL OF THE FLOW}

The common theoretical flow schemes of the RI centrifugal pumps are based on the principle of continuous flow. An example of this approach is the Euler jet theory, based on the assumption of flow in the RI with an infinite number of vanes of zero thickness and homogenous velocity and pressure fields in the inter-vanes spaces. In this case, the correction for the real process and the transition from the head pressure with an infinite number of vanes to the actual head pressure is performed by applying empirical coefficients.

The real overview of the flow in the rotary impellers of centrifugal pumps is much more complex, and also depends on the inter-influence of different energy zones. Irregularity in the energy distribution in the spatial stream is a generator of secondary flows and vortex structures. At first, they can be interpreted as the result of the interaction of local flow zones with different levels of total energy.

Numerous studies have shown that the flow along the inter-vane channels of the impeller of a centrifugal pump has a discernible spatial character with an irregular field of velocities and pressures.

The ABCS pumps belong to the class of lowconsumption superchargers. The decrease in the flow rate of the working fluid through them, with a constant coefficient of speed, is accompanied by miniaturization of the structure and an increase in the thickness of the boundary layer.

The paper [6] presents a model of the flow structure in the inter-vane channel of a centrifugal pump. According to this paper, the flow in the boundary layer is called the "secondary" flow.

The term "secondary" indicates that the flow occurs along the end wall of the RI at an angle to the (main) flow. The secondary flow reaches the back of the vanes of the RI and moves towards the average height of the cross-section of the inter-vane channel, which is facilitated by the direction of rotation of the channel vortex. In the core of the flow, there is a compensatory motion of the liquid in the opposite direction, forming a paired vortex. According to the generally accepted classification of flows, such structures are qualified as the vortex ones.

The energy side effects of a system of vortices in the inter-vane channels of the rotary impeller of a centrifugal pump are energy losses which are proportional to the level of their intensity, as well as a decrease in the pressure qualities of the pump.

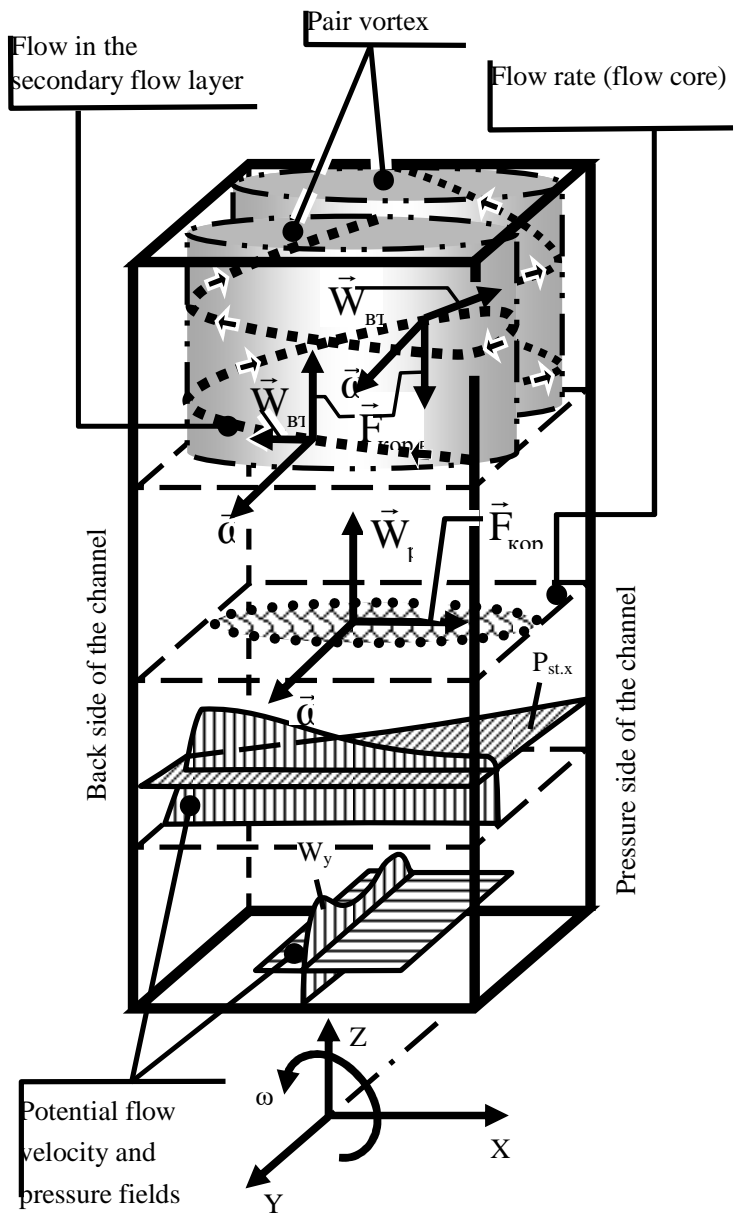

Figure 2

Besides the vortex pair, the model of the flow structure in the inter-vane channel of the centrifugal pump, mentioned in the paper [6], contains the distribution of the relative rate $\mathrm{W}$ and static pressure $\mathrm{P}_{\mathrm{st}}$ for 2 cases of flow representing, which are the following (see Fig. 2):

- potential, with the parameter identifiers $\mathrm{W}_{\mathrm{x}}, \mathrm{W}_{\mathrm{y}}$, $\mathrm{P}_{\text {st.x }}, \mathrm{P}_{\text {st. } y}$;

- viscous, consisting of a boundary layer in which secondary flows occur at a speed of $\overrightarrow{\mathrm{W}}_{\text {вт.т }}$ and 
the flow rate zone with a speed of $\overrightarrow{\mathrm{W}}_{\mathrm{pт}}$ where the index "вт.т." (s.f.) means secondary flow, index "рт" - (f.r.) flow rate. The Coriolis force $\overrightarrow{\mathrm{F}}_{\text {кор }}$ is denoted as $\overrightarrow{\mathrm{F}}_{\text {кор вт. т }}$ and $\overrightarrow{\mathrm{F}}_{\text {кор рт }}$, depending on the zone of flow -secondary or discharge, respectively. The symbol $\vec{\omega}$ denotes the vector of the angular velocity of the RI rotation.

Thus, the theoretical model of the flow structure in the inter-vane channel of the centrifugal pump allows us to draw the conclusion that the erythrocytes, passing through the fields of velocity gradients and pressures formed by the vortex flow structures, as well as the inertial forces, undergo a force action having a hydrodynamic nature of origin. To verify empirically this statement, an experiment was conducted to visualize the flow structure in RI.

\section{THE EMPIRICAL MODEL OF THE FLOW}

The study of the pattern mechanism of structured fluid flows in the channels of hydraulic pumps requires sophisticated hydrodynamic experiments. The obtained data allow us to clarify the theoretical model of the flow. This approach is fully observed in the study of the hydrodynamics of flows in the RI channels of the centrifugal pump.

This section presents the results of visualized studies of the spatial structure in the impellers of centrifugal pumps with a diameter $\mathrm{d}_{2}=41.5 \mathrm{~mm}$. To confirm the influence of the boundary layer on the flow in the RI channels, the evolution of different-energy flow zones was analyzed. These, in particular, were attributed to the separated zone, secondary flows and angular vortex.

The obtained data were essential to assess adequately the significance of the studied hydrodynamic factors, the degree of their influence on hemolysis in centrifugal pumps of the ABCS.

To detail the spatial structure of the flow in the RI under the given modes of consumption $\dot{\mathrm{V}} / \omega=(3 \ldots 3.8) \cdot 10^{-7} \mathrm{~m}^{3}$, where $\dot{\mathrm{V}}$ is the volume flow rate of the working fluid $\left(\mathrm{m}^{3} / \mathrm{s}\right), \omega-$ angular frequency of the RI rotation $\left(\mathrm{s}^{-1}\right)$, it was carried out the visualization of the flow pattern. It was used the method of flushing the indicator film in the surface layer along the main and cover discs of the RI during the pump operation. An illustration of the results is shown in Fig. 3.

The trials were carried out at the number of rounds of the impeller $n=6000$ rounds per minute. During the visualized studies the working fluid was water.

In the analysis of flow patterns, the following interpretation of the image was applied. The areas of the indelible indicator were considered as zones of complete separation of the flow. Flow zones, in which the separate current fluid lines were visualized, were identified as the areas of secondary flows, and the places of complete flushing of the indicator - as zones of the existence of inlet and angular vortexes.

\section{RESULTS}

The analysis of the obtained visualized examples showed that in addition to the pair vortex caused by the secondary flows along with the RI disks, there is a flow separation and an angular vortex, see Fig. 3. These vortex structures, according to the classification of the theory of vortex motion, belong to the screw vortices or spiral vortices resembling a tube with thickened walls. The existence of vortex structures is accompanied by pressure pulsations and self-oscillations [7-10]. Any solid particle or cell filled with cytoplasm, getting into the vortex structure, begins to rotate along a spiral path, undertaking the action of the centrifugal force of inertia and the pulse drop of static pressure in the form of a kind of hydraulic hammer. The larger the size and mass of the cell, the greater the force it will undertake, being in the vortex.

\section{CONCLUSION}

It is a very high probability that the destruction of red blood cells in the centrifugal pump of the artificial blood circulation system occurs during their passage through the vortex flow structures occurring in the inter-vane channels of the impeller of the centrifugal pump.

It is necessary to continue theoretical and experimental research in this area to prove or disprove this conclusion, based on the indirect data rather than direct ones. 

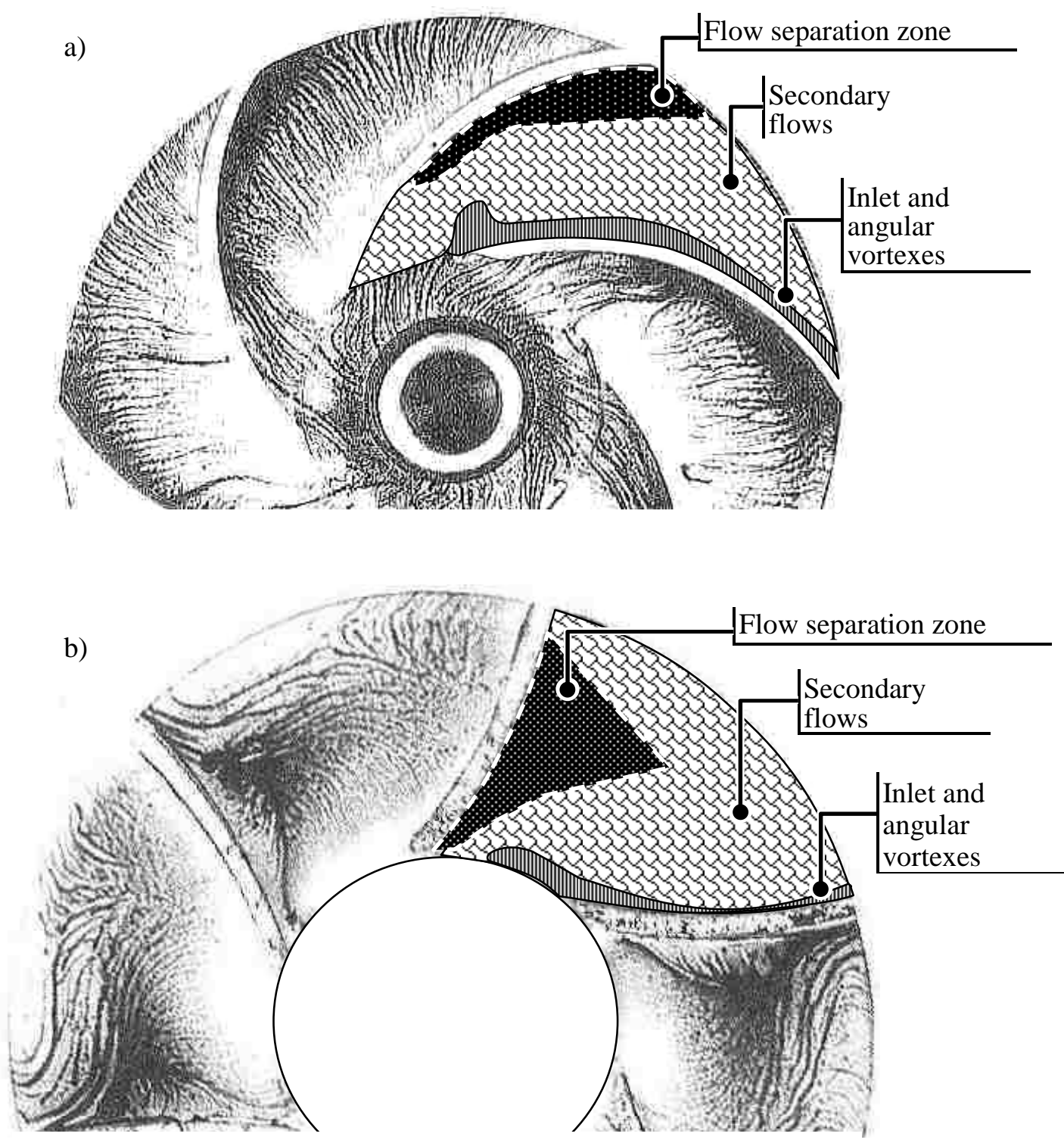

Figure 3 Identification of the flow structure in the boundary layer on the main and cover disks of the RI: a - the main disc, the angle of the RI vanes at the outlet of $30^{\circ} ; \mathrm{b}-$ the cover disc, the angle of the RI vanes at the outlet of $90^{\circ}$

\section{REFERENCES}

[1] A.M. Chernyavskiy, A.V. Fomichev, T.M. Ruzmatov et al., Prospects of Disk Pump for Mechanical Circulatory Support in Cardiac Surgery (Review), Bull. of Transplantat. and Artificial Organs XVIII/3 (2016) 69-73.

[2] V.I. Shumakov, G.P. Itkin, O.V. Romanov et al., Modern Problems of Mechanical Support of Blood Circulation, Mechatronics, Automat., Control 8 (2007) 34-40.

[3] R.B. Medvitz, A.D. Boger, V. Izraelev et al., CFD Design and Analysis of a Passively Suspended Tesla
Pump Left Ventricular Assist Device, Artificial Organs 35(5) (2010) 522-533.

[4] M.V. Kraev, V.A. Lukin, B.V. Ovsyannikov, Low Energy Pumps of Aerospace Systems, 1985, 128 p.

[5] D.J. Laser, J.G.A. Santiago, Review of micropumps, J. of Micromechan. and Microengineer. 14(6) (2004) $35-64$.

[6] B.V. Ovsyannikov, A.V. Bobkov, Geometric Model of Flow Stream in Small-Sized Wheels of Centrifugal Pumps, Scientific and technical issues of modern hydraulic engineering and methods of their solution, Collected Papers of International Scientific 
and Technical Conference, Nestor, St. Petersburg, 2001, pp. 49-53.

[7] D.V. Knyazev, Localized Convective Vortices in a Viscous Fluid, Convective Flows 2 (2005) 17-25.

[8] V.F. Kopiev, M.Yu. Zaitsev, L.P. Guriashkin, V.A. Yakovlev, A Technique for Visualization of the Turbulent Vortex Ring, Atlas of Visualizat. 2 (1996) 139-149.
[9] V.N. Blazhko, S.G. Chefranov, On Self-Oscillations Occurring at the End of a Swirling Jet, Proc. of the Russ. Acad. of Sci. Fluid mechan. 5 (2005) 99-106.

[10] S.G. Chefranov, Generation of Helicity in Homogeneous Helical and Vortex Flows, Proc. of the Russ. Acad. of Sci. J. of Experim. and Theoret. Phys. 126(5) (2004) 1133-1145. 